Meta

Journal des traducteurs

Translators' Journal

\title{
Dynamique historique des (re)traductions du Quijote en français : questions méthodologiques et premiers résultats
}

\section{Clara Foz et María Sierra Córdoba Serrano}

Volume 50, numéro 3, août 2005

Le prisme de l'histoire

The History Lens

URI : https://id.erudit.org/iderudit/011613ar

DOI : https://doi.org/10.7202/011613ar

Aller au sommaire du numéro

Éditeur(s)

Les Presses de l'Université de Montréal

ISSN

0026-0452 (imprimé)

1492-1421 (numérique)

Découvrir la revue

Citer cet article

Foz, C. \& Córdoba Serrano, M. (2005). Dynamique historique des (re)traductions du Quijote en français : questions méthodologiques et premiers résultats. Meta, 50(3), 1042-1050. https://doi.org/10.7202/011613ar
Résumé de l'article

Après avoir présenté un bref état de la question touchant aux traductions françaises du Quijote de Cervantes, nous présentons la base de données que nous sommes en train de construire et qui nous permettra de tracer la dynamique de l'ensemble des (re)traductions françaises du Quijote, depuis la première publiée en 1614 par César Oudin à la dernière, publiée sous la direction de Jean Canavaggio dans la collection La Pléiade en 2001. Nous analysons les possibilités méthodologiques offertes par la base et proposons un premier aperçu de ses applications possibles. 


\title{
Dynamique historique des (re)traductions du Quijote en français: questions méthodologiques et premiers résultats
}

\author{
CLARA FOZ \\ Université d'Ottawa, Ottawa, Canada \\ cfoz@uottawa.ca \\ MARÍA SIERRA CÓRDOBA SERRANO \\ Université d'Ottawa, Ottawa, Canada
}

\section{RÉSUMÉ}

Après avoir présenté un bref état de la question touchant aux traductions françaises du Quijote de Cervantes, nous présentons la base de données que nous sommes en train de construire et qui nous permettra de tracer la dynamique de l'ensemble des (re)traductions françaises du Quijote, depuis la première publiée en 1614 par César Oudin à la dernière, publiée sous la direction de Jean Canavaggio dans la collection La Pléiade en 2001. Nous analysons les possibilités méthodologiques offertes par la base et proposons un premier aperçu de ses applications possibles.

\section{ABSTRACT}

After a brief look at the existing resources on the French translations of Don Quixote by Cervantes, we introduce the data base that we are presently building in order to trace its dynamics. This base will treat all French translations, from the first one, published in 1614 by César Oudin, to the most recent one, prepared under the direction of Jean Canavaggio for the La Pléiade collection in 2001 . We analyze the methodological assets that such a base offers and show briefly what applications could be derived from it.

\section{MOTS-CLÉS/KEYWORDS}

histoire de la traduction, méthodologie de l'histoire de la traduction, traductions du Quijote en français

\section{État de la question}

L'année 2005 commémore le 400e anniversaire de la publication du Quichotte en Espagne $^{1}$ et s'annonce marquée par une recrudescence des activités et des célébrations consacrées aux aventures du célèbre chevalier. Les «spécialistes » se réuniront dans le cadre de très nombreux congrès, colloques ou rencontres. Il y a même fort à parier que les touristes voyageant dans la région de la Manche se verront proposer, plus que jamais sans doute, des menus aux accents quichottesques et des promenades in $s i t u^{2}$. Sur la Toile, le Quichotte est plus présent que jamais et un portail consacré à cet anniversaire $^{3}$ est venu s'ajouter aux nombreuses pages existant sur l'auteur et son œuvre. Ce portail, créé par la Fundación Biblioteca Virtual Miguel de Cervantes, présente essentiellement des textes cervantins, une importante iconographie ainsi qu'un calendrier d'événements associés au quatre centième anniversaire de la publication du Quijote. Fait nouveau et remarquable, la traduction y occupe une place non négli- 
geable: sont proposées en ligne un certain nombres de traductions, dont le fac-similé de la première traduction française, proposée par César Oudin en 1614. Les versions dans d'autres langues, fondamentales dans la réception de cette ouvre à laquelle convient parfaitement la dénomination $\mathrm{d}^{\prime}$ " $\operatorname{archétexte}^{4} »$, y tiennent le haut du pavé. Ce fait est particulièrement frappant si l'on compare cette page à celle du Cervantes Project ${ }^{5}$ : en effet, dans le cadre de cette initiative, institutionnelle, tout comme pour la précédente, la traduction (ses produits tout comme ses acteurs) se voit réduite à la portion congrue 6 . Faut-il s'en étonner? En effet, si l'œuvre majeure de Cervantes a fait couler tellement d'encre qu'il apparaît parfois plus facile de dénombrer les «grands auteurs» n'ayant jamais fait allusion au "premier grand roman moderne» que ceux qui en ont d'une manière ou d'une autre parlé, en revanche elle n'a guère attiré le regard des traductologues ou plus largement des chercheurs que l'histoire de la traduction intéresse. Il faut dire que les catégories qui ont permis de répertorier, analyser et expliquer les faits de traduction ont pendant longtemps été d'ordre essentiellement pédagogiques ou théoriques et se trouvent le plus souvent tournées vers le texte lui-même et son transfert d'une langue à l'autre. Notre propos à nous relève d'un choix délibéré de prendre des distances par rapport aux études entièrement tournées vers le texte lui-même: nous savons en effet que le traduire se définit davantage par ce qu'il fait que par ce qu'il est. Notre but, en dégageant le schéma dynamique des traductions françaises du Quijote, est de faire ressortir un certain nombre de tendances, mais également de montrer les vides, de laisser apparaître des limites culturelles ou politiques, de déterminer les périodes de retraduction active et celles au cours desquelles la retraduction est passive, d'analyser, à travers la production et la réception des traductions, les différents statuts dont est investie selon les époques l'œuvre de Cervantes : livre d'érudition, manuel pour hispanophiles, livre à raconter de vive voix, album illustré destiné au jeune public ou livre-cadeau «présentoir» des gravures de Gustave Doré.

\section{Questions méthodologiques}

Ce travail sur la ou les fonctions historiques et sociales des traductions impliquent que soient réunies des données empiriques sur l'objet à l'étude et donc que soit construite une base de données d'une part interrogeable, mais également adaptable et susceptible d'être revue et mise à jour en fonction de découvertes bibliographiques. Tout travail bibliographique suppose en effet un système de références ouvert et non définitif. La base de données, construite en mode Access, se présente sous la forme de deux tables: la première s'intitule Éditions (nous reviendrons sur ce terme) et la seconde regroupe des renseignements sur les agents eux-mêmes, les acteurs; elle a été baptisée Traducteurs (Aline Schulman constitue à notre connaissance la seule traductrice du Quijote en français) ${ }^{7}$. Les catégories comprises dans la première table présentent tout un ensemble de données éditoriales qui vont de la date de publication, au format, au genre (collections destinées aux enfants ou aux bibliophiles par exemple), en passant par la présence ou non d'une préface, d'une vie de Cervantes, d'une notice sur l'auteur de la traduction, de notes du traducteur et d'illustrations, entre autres choses. Au total, cette partie de la base se fonde sur quarante et un champs, parmi lesquels figure un champ dénommé "Commentaires», qui permet d'inscrire les points d'interrogation pouvant surgir de cette masse de données. C'est une catégorie 
particulièrement utile et nécessaire et qui permet de noter les doutes, les difficultés et les hésitations dont il convient de garder trace dans la mesure où la résolution de ceux-ci peut survenir à une étape ultérieure, face à une difficulté comparable, par exemple. L'objet qui nous intéresse, à savoir l'ensemble des éditions en français toutes époques (soit de 1614 à 2001) et traducteurs confondus, implique le regroupement et la manipulation d'un grand nombre de données bibliographiques concernant les textes ainsi que les traducteurs, ce qui n'est pas sans entraîner un certain nombre de difficultés.

\section{Problèmes d'ordre général}

Une des difficultés rencontrées, connue de tous ceux qui s'intéressent à l'histoire de la traduction avant, en gros, les années 1950, c'est la rareté ou l'absence de bibliographies propres au domaine: la situation varie bien entendu selon les pays et les époques et l'Amérique latine dispose d'une longue tradition bibliographique par rapport à ce qui se passe pour le domaine français. Les données contenues dans notre base sont extraites d'une bibliographie publiée dans les années 1930 par les presses de l'Université Harvard (Ford et Lansing 1931) et de deux catalogues principaux: celui de la Bibliothèque nationale de France (BNF) et celui de la Biblioteca Nacional d'Espagne (BNE). Les données fournies par Ford et Lansing seront comparées à celles des catalogues de la BNF et de la BNE pour la période allant jusqu'à 1929 (date à laquelle prend fin le travail de Ford et Lansing). D’autres vérifications seront menées sur la base d'autres bibliographies ou catalogues limités dans le temps ou l'espace ${ }^{8}$.

Les autres limites d'un travail de ce genre sont représentées par le fait que la base étant construite en quelque sorte manuellement, des erreurs sont possibles, pour ne pas dire normales. Mais les risques d'erreur dans l'alimentation de la base sont également liés à la qualité plus ou moins grande des catalogues auxquels nous puisons. Il se trouve par exemple que les entrées correspondant à la Biblioteca Nacional d'Espagne pour les années 1900 à 1950 sont particulièrement problématiques du fait que nombre d'éditions ne présentent aucune date et ont été abusivement datées «1900». Par ailleurs, comme nous avons pu le constater et comme nous l'ont confirmé des sources émanant de la BNE, la qualité de la saisie des fiches manuelles présente également un certain nombre de limites: il arrive par exemple qu'une mention comme celle de «édition destinée à l'enfance» fasse l'objet de déformations tout à fait étonnantes, si elle est transcrite par quelqu'un qui ignore le français ou n'en possède que quelques rudiments. Les risques de doublets sont également considérables sur la question du format: il convient d'être particulièrement attentif aux variations dans la présentation matérielle, dans le format essentiellement, lorsque cette information-là seulement varie pour une entrée donnée. Il est clair que seul l'examen minutieux des exemplaires permet parfois de déterminer si nous avons affaire à des éditions différentes ou pas. Ainsi, les premiers résultats risquent de présenter un bruit documentaire considérable qui ne devrait cependant pas infirmer les tendances générales observées, mais qui devra être éliminé au fur et à mesure que la base sera retravaillée et les données qu'elle contient, individuellement vérifiées.

La question se pose également de savoir comment définir le terme d'éditions. Comme l'indique très justement José Manuel Losada Goya dans la bibliographie à laquelle nous avons fait référence précédemment: 
Ce qui peut sembler superfétatoire de prime abord ne l'est pas une fois que l'on a collationné les nombreux tirages d'une même œuvre, si semblables entre eux qu'ils ne varient que par le nom du libraire ou leur date; il est nécessaire alors de distinguer entre les vraies rééditions, les contrefaçons et les simples réimpressions (généralement, dans ces dernières seule la page du titre était modifiée (1999: xi).

Le problème est particulièrement crucial pour ce qui est des traductions dans la mesure où, suivant les époques, d'une part, le nom de l'auteur et du traducteur n'apparaissent pas forcément ou dans un ordre préétabli et, d'autre part, du fait qu'il s'avère souvent malaisé de tracer clairement une ligne de démarcation entre traductions nouvelles, rééditions d'une traduction connue ou existante ou réélaboration adaptation basée sur une, voire plusieurs traductions. C'est dans l'optique de tenter de démêler quelque peu l'écheveau bibliographique devant lequel se retrouve le chercheur que nous avons eu recours à la notion de "traduction matricielle», notion qui renvoie pour nous à toute traduction ayant donné lieu à un nombre élevé de réélaborations, adaptations, versions revues et corrigées. Peuvent être qualifiées de matricielles les traductions d'Oudin (1614), de Rosset (1618), de Filleau (1677), de Florian (1798) et de Viardot (1836). De celles-ci, on peut dire qu'elles ont donné lieu, sous une forme ou une autre, à de nombreuses rééditions (telles quelles ou revues et corrigées). Les chiffres correspondant à ces traductions considérées comme telles pourront donc varier par rapport à ceux qui renvoient aux différents traducteurs. Cette question permet d'ailleurs d'aborder celle du sujet «traducteur» : pour tracer le schéma historique des traductions françaises du Quijote, il nous semble que l'accent sur l'agent lui-même, soit le traducteur, est de moindre importance. Certes, nous disposons d'une table consacrée aux traducteurs et il ne sera pas sans intérêt d'en dégager un certain nombre de tendances. Les traducteurs français du Quijote ont-ils en général traduit d'autres auteurs et lesquels? Ont-ils répondu à une commande, un commanditaire? Traduit pour d'autres raisons et lesquelles? Étaient-ils animés du souci de renouveler le domaine? Ont-ils traduit les deux parties l'une à la suite de l'autre? Quelle est la proportion de ceux qui ont déclaré se baser sur une version antérieure? Quels liens reconnaissent-ils entre leur version et celle(s) qui précède(nt)?

L'ensemble des données contenues dans la base sera analysé d'un point de vue systémique, comme appartenant à un système global délimité dans le temps. Les observations qui seront tirées de celle-ci permettront de parvenir à une vision d'ensemble des retraductions françaises du Quijote et de déterminer un certain nombre de tendances selon les périodes observées (un siècle, cinquante ans, une décennie, voire un temps historique marqué par un ou plusieurs événements, qu'il s'agisse du renversement symbolique et diplomatique liée à la Paix des Pyrénées (Schaub 2003 : 11) ou d'événements plus sanglants comme les invasions napoléoniennes ou la guerre civile espagnole. Bien entendu, tant la manière de classer les données, d'ordonner la connaissance que les façons d'interroger la base auront des répercussions sur les réponses obtenues, cela va sans dire. Aucune recherche de type quantitatif ne peut en effet être qualifiée de neutre, d'objective, aucune donnée n'est transparente.

\section{Problèmes particuliers}

Le corpus à partir duquel est composé la base exige que soit clairement défini ce que l'on entend par traduction. Les critères de sélection doivent être déterminés en fonction 
des objectifs généraux et spécifiques de la recherche: faut-il considérer qu'un ouvrage présentant un Quichotte en 122 pages constitue encore une traduction de l'œuvre de Cervantes? En d'autres termes, le critère du nombre de pages doit-il entrer en ligne de compte? Nous sommes d'avis que non. Il se trouve en effet qu'à certaines époques le Quijote a été présenté au public français surtout sous une forme abrégée. Or la prise en compte de ces ouvrages permet précisément de reconstruire les différentes manières dont l'œuvre a été (re)présentée au public français, ou à un certain public français, puisque les horizons d'attente ont varié selon les époques et les publics. Nous avons en revanche exclu a priori tous les textes qui se présentent comme des imitations: il s'agit le plus souvent d'épisodes particuliers, de saynètes ou de pièces de théâtre basés sur l'œuvre de Cervantes et qui s'en inspirent dans un but de valorisation ou de subversion. Une autre difficulté de taille dans ce domaine est représentée par le manque d'uniformité des entrées et de la saisie des informations bibliographiques, en particulier dans la manière dont est consigné le titre des traductions. Là encore, une analyse serrée permettra de dégager un certain nombre de tendances susceptibles de contribuer à définir le schéma des (re)traductions du Quijote en français: sont en effet d'emblée détectables dans la traduction des titres diverses tendances déformantes, pour reprendre la terminologie employée par Berman. Mais un titre demeure un titre et les rapports pouvant être établis entre les tendances observables dans celui-ci et les stratégies à l'œuvre dans la traduction méritent également d'être considérés avec la plus grande attention.

\section{Premiers résultats}

Les applications possibles auxquelles une base de données comme celle que nous venons de décrire succinctement peut donner lieu sont multiples. Nous en proposerons trois: la première (voir graphique 1) présente une répartition par traducteur qui couvre la période 1614 (date de la première traduction) à 1850, période pour laquelle nos données sont particulièrement fiables et peuvent, dans toute la mesure du possible, être considérées comme définitives.

À chaque traducteur est associée une date, qui correspond à la première date de publication de sa traduction. On voit que pour cette période ce sont les traductions d'Oudin et de Filleau qui donnent lieu au plus grand nombre de publications. Pour Oudin, il faut nuancer ou plutôt préciser qu'à partir de 1639 les deux parties de l'œuvre sont réunies en français. Il s'ensuit que Rosset (premier traducteur de la seconde partie) n'obtient pas toujours la place qui lui revient, l'ensemble de l'œuvre étant parfois attribué à Oudin seulement. Bien entendu, il s'agit de données qui demandent à être analysées, précisées et mises en parallèle avec d'autres. Ainsi, si l'on compare les chiffres obtenus par traducteur avec les chiffres totaux d'éditions par siècle (graphique 2), on s'aperçoit qu'un nombre conséquent de publications omettent de mentionner le nom du traducteur, ne le précisent pas, ou éventuellement que l'identité du traducteur ne figure pas dans les renseignements consignés sur les fiches bibliographiques. Et ce genre de renseignement est intéressant, en soi. Le graphique 2 auquel nous venons de faire allusion présente quant à lui une répartition du nombre des éditions publiées par siècle. La comparaison est malaisée dans la mesure où les siècles sont ici complets, contrairement au premier graphique qui ne donne des chiffres que jusqu'à 1850. La progression est nette d'un siècle à l'autre, même si nous 
devons reconnaître, comme nous l'avons déjà mentionné, que pour le XIX ${ }^{\mathrm{e}}$ siècle nos données pourraient présenter un bruit documentaire non négligeable: nous n’avons pas encore affiné les résultats obtenus et la présence d'un certain nombre de doublets est probable. La tendance générale, cependant, ne devrait nullement s'en trouver renversée. Le dernier graphique se décompose en trois pour les trois siècles auxquels il est fait référence $\left(\mathrm{XVII}^{\mathrm{e}}, \mathrm{XVIII}^{\mathrm{e}}\right.$ et $\left.\mathrm{XIX}^{\mathrm{e}}\right)$ et donne une indication des lieux de publication des éditions françaises. C'est à une analyse fondée sur les rapports économiques et culturels entre l'Espagne et la France ainsi que les autres pays dont il est ici question. Ces données devront être comparées à d'autres tendances observables dans le monde de l'édition. Nous espérons aussi qu'elles contribueront à éclairer les rapports entre l'Espagne et la France aux diverses époques concernées.

On peut espérer avec ce genre de recherche voir la traductologie contribuer à enrichir d'autres disciplines comme l'histoire: dans son sens le plus général, mais aussi l'histoire des mentalités, du livre ou de la lecture; des représentations aussi, par la manière dont les traductions rendent compte des relations culturelles et politiques en général et contribuent à les forger, ou voire parfois les contrarient. Le phénomène ou l'objet traduction ainsi envisagé ne saurait être autre chose qu'une des formes de la représentation, à l'instar de l'architecture, des œuvres d'art ou du théâtre, non pas unique en tant que telle, mais comme élément d'un ensemble de représentations culturelles et sociales qui produisent du sens, un sens. Envisagées dans le cadre du «système littéraire» français, qui a partie liée avec les relations culturelles et politiques, les questions entourant la traduction, loin de se limiter aux relations entre un texte source et un texte cible, sont observées comme une sorte de baromètre des relations franco-espagnoles, un témoin des discours entourant celles-ci. Ce qui pourrait éventuellement mener à inverser certains termes, certains rapports, et à montrer que les traductions ne constituent pas forcément un mouvement d'une culture réceptrice qui s'alimenterait à une culture donatrice, que les rapports instaurés par cette pratique sont beaucoup plus complexes et dépassent largement ceux d'un «original» avec des textes qui lui seraient secondaires, ceux d'un auteur du passé avec ses traducteurs ou ses lecteurs.

\section{NOTES}

1. Rappelons que c'est le 16 janvier 1605 que sortait des presses la première partie du Quijote.

2. Tout particulièrement dans la petite bourgade de Villanueva de los Infantes située à 220 kilomètres au sud de Madrid, lieu dont Cervantès aurait, semble-t-il, préféré cacher le nom.

3. Il se trouve à l'adresse suivante: <http://www.cervantesvirtual.com/IVCentenario/>.

4. Il s'agit des « œuvres qui ont un statut exemplaire, qui appartiennent au corpus de référence d'un ou plusieurs positionnements d'un discours constituant. La notion est de D. Maingueneau et F. Cossutta (1995).

5. <www.csdl.tamu.edu/cervantes/english/>.

6. Dans la bibliographie en ligne (Cervantes International Bibliography Online: CIBO) sous la rubrique Translations figure une seule entrée consacrée à une série de traductions. On trouve ailleurs dans la bibliographie des références à diverses traductions ainsi qu'un nombre limité d'articles portant sur ce thème, mais il n'en demeure pas moins que la rubrique Traducciones est réellement squelettique.

7. On observera qu'une des dernières traductions publiées en anglais est également l'œuvre d'une femme, Edith Grossman, de fait qualifiée par Harold Bloom du «Glenn Gould de la traduction »... du fait qu'elle articule chaque note! (Grossman 2003: xxii).

8. Par exemple, l'excellente Bibliographie critique de la littérature espagnole en France au XVII siècle de José Manuel Losada Goya (1999). 


\section{RÉFÉRENCES}

Bardon, M. (1931): Don Quichotte en France aux XVII et XVIII ${ }^{e}$ siècles 1605 - 1805, Paris, Librairie ancienne Honoré Champion, Tome 1.

Biedermann, F. (1837): Don Quichotte et la tâche de ses traducteurs [ Observations sur la traduction de Viardot accompagnées d'éclaircissements nouveaux dans le style et l'interprétation de l'original et dans l'esprit de son auteur], Paris, Librairie de Delaunay.

Bloom, H. (2001) (ed): Cervantes's Don Quixote. Modern Critical Interpretations, Philadelphia, Chelsea House Publishers.

CADERNOS DE TRADUÇAO (2003): «Traduçâo, retraduçâo e adaptaçâo» Milton, John et Marie-Hélène Catherine Torres (ed), Univeridade Federal de Santa Catarina, Brésil.

Cervantes, M. DE (1884 [ 1614 et 1618 ]): L'histoire de Don Quichotte de la Manche, première traduction française par C. Oudin et F. de Rosset, avec une préface par Émile Gebhart, dessins de J. Worms gravés à l'eau-forte par De Los Ríos, 6 vol., Paris, Librairie des bilbliophiles.

Cervantes, M. DE (1949 [1934]) : L'ingénieux hidalgo Don Quichotte de la Manche, trad. par César Oudin et François Rosset, revue, corrigée et annotée par Jean Cassou; [suivi de] Nouvelles exemplaires, traduction et notes de Jean Cassou, coll. «Bibliothèque de la Pléiade», Paris, Gallimard.

Cervantes, M. De (1997): L'ingénieux hidalgo don Quichotte de la Manche, trad. par Aline Schulman, préface de Jean-Claude Chevalier, 2 volumes, Paris, Éditions du Seuil.

Cervantes, M. De (2001): Euvres romanesques complètes, trad par Jean Canavaggio, Claude Allaigre, Michel Moner, Jean-Marc Pelorson, 2 volumes, coll. «Bibliothèque de la Pléiade», Paris, Gallimard.

Charaudeau, P. et D. Maingueneau (2002): Dictionnaire d'analyse du discours, Paris, Éditions du Seuil.

Flores, A. et M.J. Bernadete (1969) (ed): Cervantes through Centuries, Gordian Press.

Ford, J. D.M. et R. LANsing (1931): Cervantes: A tentative Bibliography of his Works and of the Biographical and Critical Material Concerning Him, Cambridge, Massachusetts, Harvard University Press.

Foz, C. (2003) : «(Re)traduction(s) et (Re)présentation(s): première et dernière sortie du Quijote en français ». Cadernos de Traduçâo n ${ }^{\circ}$ XI, 1. Universidade Federal de Santa Catarina, Brésil, p. 39-57.

Lenaghan, Patrick (2003): Imágenes del Quijote. Modelos de representación en las ediciones de los siglos XVII a XIX, Madrid, The Hispanic Society of America, Museo Nacional del Prado, Real Academia de Bellas Artes de San Fernando, Calcografía Nacional.

LOSADA Goya, J. M. (1999): Bibliographie critique de la littérature espagnole en France au XVII siècle. Présence et influence, Genève, Librairie Droz S.A.

MAGAZINE LITTERRAIRE (n 358, 1997): «Don Quichotte ou l'invention du roman moderne », Paris, p. 16-55.

Maingueneau, D. et F. Cossutta (1995) : «L'analyse des discours constituants », Langages 117, Paris, Larousse, p. 112-125.

Meschonnic, H. (1999): Poétique du traduire, Lagrasse, Verdier.

PALIMPSESTES (1990): Retraduire (nº), Paris, Publications de la Sorbonne Nouvelle.

Pérez, J. (2000): «Les rapports culturels entre la France et l'Espagne (Xvi ${ }^{\mathrm{e}}$ et $\mathrm{XVII}^{\mathrm{e}}$ siècle), Les monarchies française et espagnole (coord. Hélène Fréchet et Christian Hermann), Paris, Éditions du temps, p. 313-322.

Pyм, A. (1998): Method in Translation History, Manchester, UK, St Jerome Publishing.

Schaub, J.-F. (2003) : La France espagnole. Les racines hispaniques de l'absolutisme français, Paris, Éditions du Seuil, Collection l'Univers historique.

VAN BRAgt, K. (1989): «Corpus bibliographiques et analyses de traductions. Un Programme d'analyse par ordinateur », Revue de littérature comparée 250, Paris, Didier Érudition, p. 171178. 


\section{Ressources électroniques}

BNE: <www.bne.es/>

BNF: < www.bnf.fr >

Cervantes Project: www.csdl.tamu.edu/cervantes

IV Centenario del Quijote: http://www.cervantesvirtual.com/IVCentenario/

HISTAL: www.histal.umontreal.ca/

\section{Graphique 1}

\section{Traductions 1614-1850}

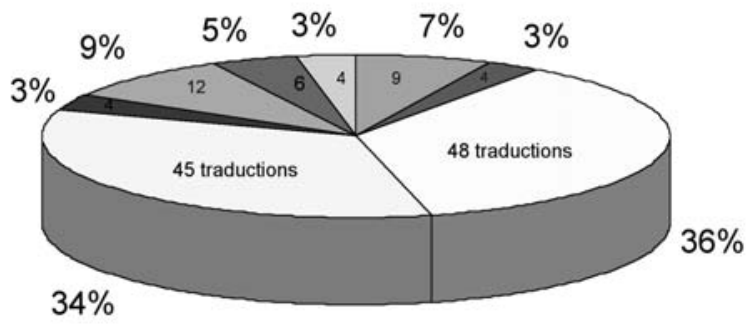

\section{Graphique 2}

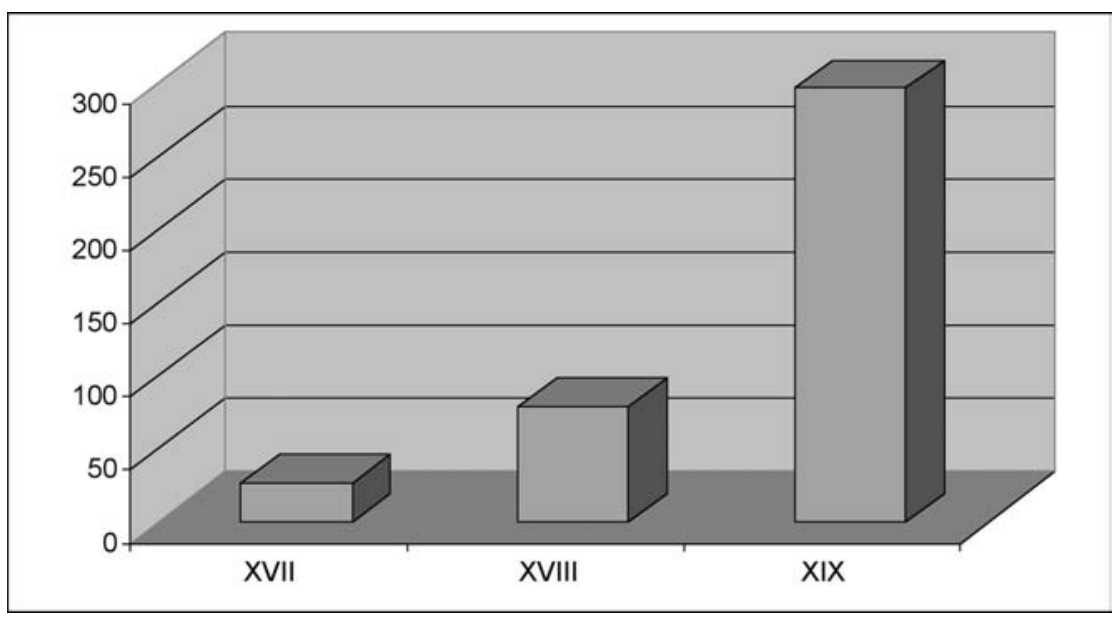

ÉDITIONS DU QUIJOTE (1614-1899)

Nombre total de publications: 402 (XVII ${ }^{\mathrm{e}}$ siècle : 27 ; XVIII ${ }^{\mathrm{e}}$ siècle: 79; XIX ${ }^{\mathrm{e}}$ siècle : 296$)$ 


\section{Graphique 3}

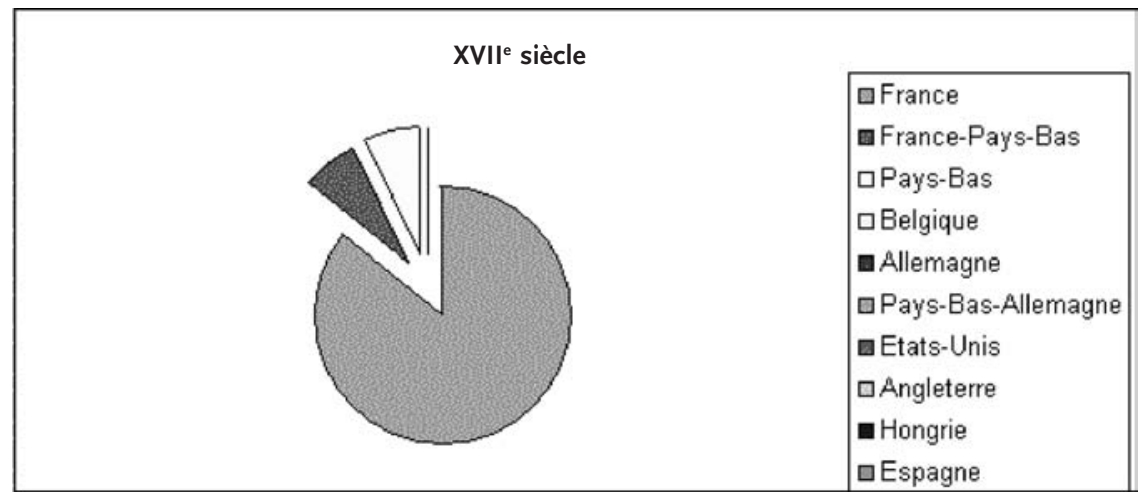

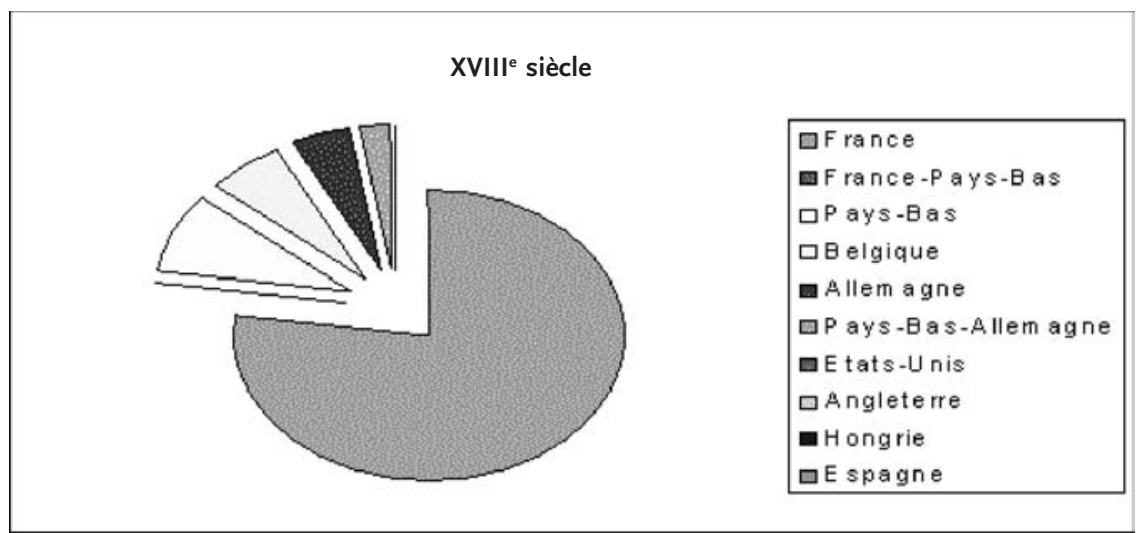

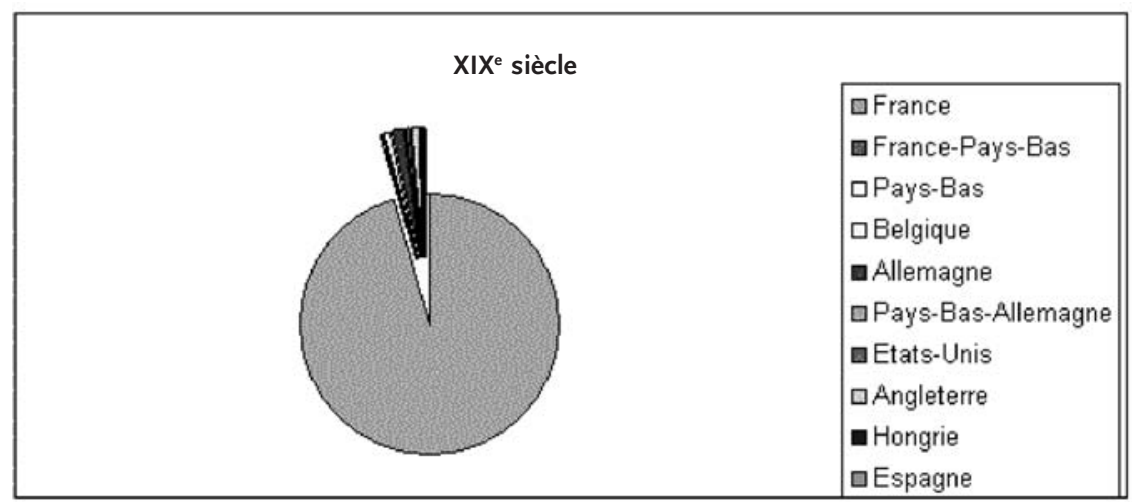

